\title{
Systematic Study of Potential False Azimuthal Asymmetries in SpinQuest
}

\author{
Forhad Hossain
}

with

Stephen Pate, Vassili Papavassiliou, and Abinash Pun

On behalf of the SpinQuest Collaboration

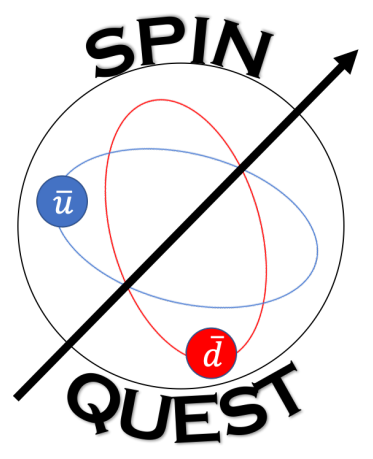

Fermilab

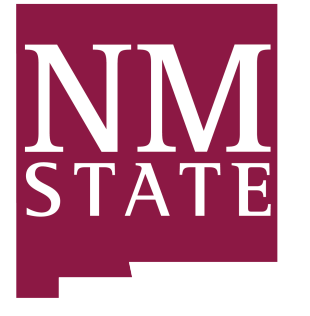




\section{Sea-quark Sivers Asymmetry from Polarized Drell-Yan}

The Drell-Yan Cross Section in Terms of Sivers Asymmetry:

$\sigma_{D Y}^{\uparrow \downarrow}=\frac{d \sigma^{L O}}{d^{4} q d \phi_{S}} \propto 1 \pm\left|\mathrm{S}_{\mathrm{T}}\right| \sin \phi_{S} A_{T}^{\sin \phi_{S}}$

Phys. Rev. D 79, 034005 (2009),

PRL 119, 112002 (2017)

$A_{n}\left(\phi_{S}\right)=\frac{1}{\left|S_{T}\right|} \frac{\sigma_{D Y}^{\uparrow}-\sigma_{D Y}^{\downarrow}}{\sigma_{D Y}^{\uparrow}+\sigma_{D Y}^{\downarrow}}=\sin \phi_{S} A_{T}^{\sin \phi_{S}}$

$x y z$ is the detector rest frame

$\vec{S}_{T}=$ Target spin vector.

$\vec{q}_{T}=$ Dimuon's transverse momentum.

$\phi_{q_{T}}=$ Azimuthal angle of the $\vec{q}_{T}$ vector.

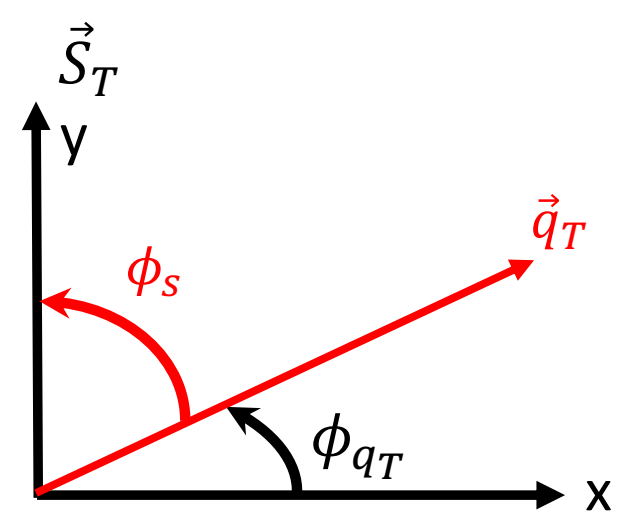

$\odot$

$+z$ is out of the page

1. $\sigma_{D Y}^{\uparrow \downarrow}$ is the Drell-Yan cross section and $A_{T}^{\sin \phi_{S}}$ is the Sivers asymmetry .

2. Azimuthal angle $\phi_{S}$ and $\phi_{q_{T}}$ can be written as $\phi_{S}^{\uparrow \downarrow}=\left( \pm \frac{\pi}{2}-\phi_{q_{T}}\right)$. 


\section{Study I: Motivation for the Spectrometer- Induced Asymmetry Systematic Check}

$$
\mathrm{A}_{\mathrm{n}}\left(\phi_{q_{T}}\right)=\frac{N_{D Y}^{\uparrow}\left(\phi_{q_{T}}\right)-N_{D Y}^{\downarrow}\left(\phi_{q_{T}}\right)}{N_{D Y}^{\uparrow}\left(\phi_{q_{T}}\right)+N_{D Y}^{\downarrow}\left(\phi_{q_{T}}\right)}
$$

$N_{D Y}^{\uparrow \downarrow}=$ The Drell Yan normalized event counts with the target polarization up (down).

The Azimuthal asymmetry can be extracted from the above formula that measures the changes dimuon yields in different spin states. The arrow $\uparrow$

$(\downarrow)$ represents the polarization up (down). We consider two possible causes for the spectrometer induced azimuthal asymmetry:

1. The efficiency of the detectors could be changing over time (e.g. the phototubes might suffer a decrease in gain).

2. The numbers of protons hitting the polarized target will not be the same in each spill, and the detector performance could change due to luminosity fluctuations.

$\rightarrow$ In either case, it is possible for one target spin state to collect a higher yield than the other, producing a false asymmetry. We will check the size of such effects, by using the SeaQuest experiment dataset. 


\section{Study II: Motivation for the Beam-offset Study}

1. Since the single spin asymmetries are extracted from different trigonometric fitting functions in the azimuthal modulations, reconstructing the dimuon momentum $q_{T}$ correctly is very important to get correct reconstructed azimuthal distributions.

2. If there was any offset in the beam and target interaction point, and if we did not know the offset information, then the dimuon phi distributions could be changed or distorted.

$\rightarrow$ We will be investigating the effect of the offset to the dimuon distributions and evaluate if they can produce any asymmetry. 


\section{Introduction to the Beam-Offset: An Illustration}

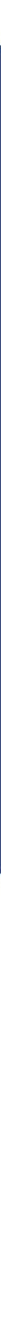

In this study we have set the offset in the Y direction 


\section{Introduction to the Beam-Offset : Accepted True Dimuons}

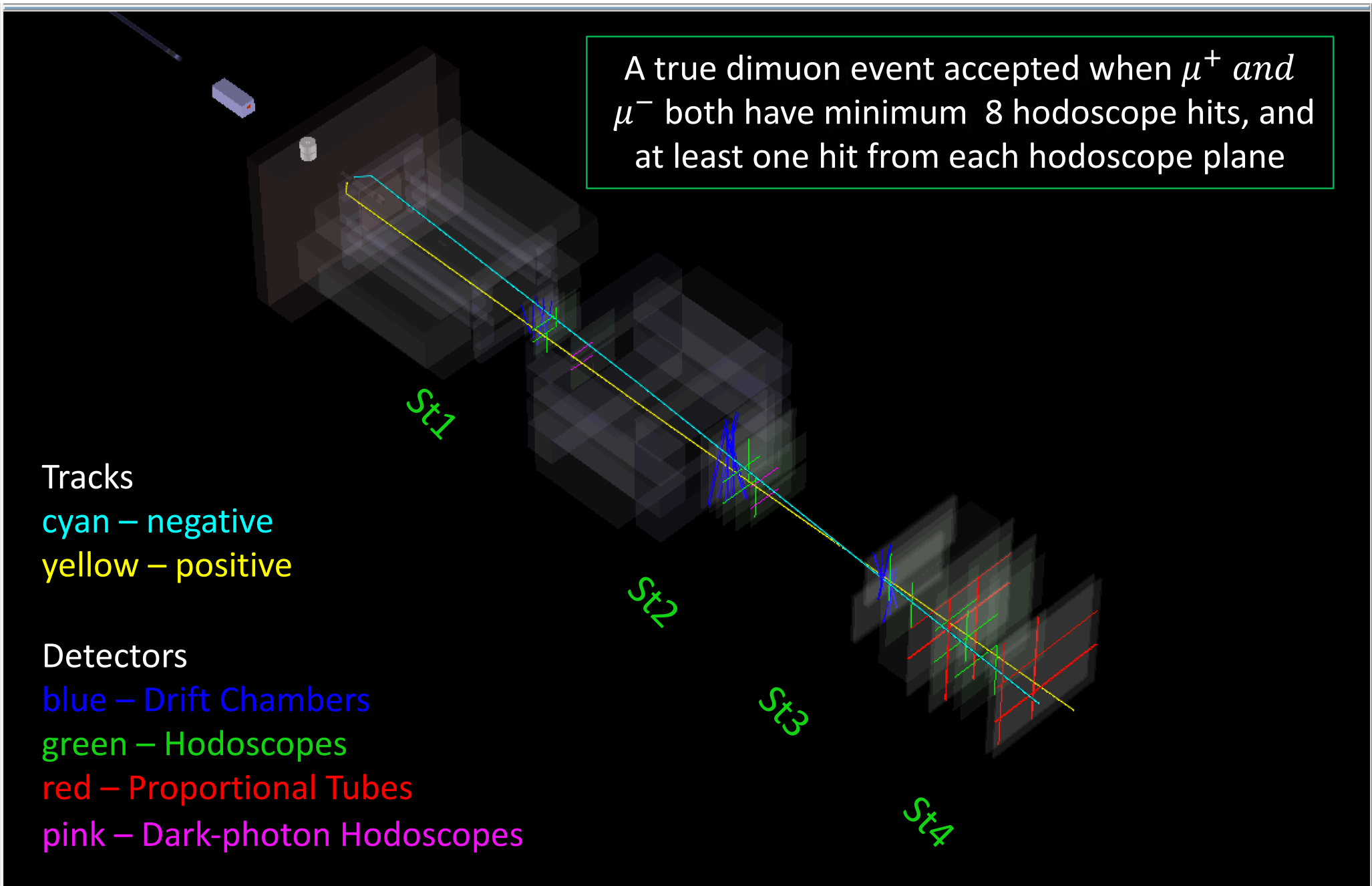




\section{Azimuthal Distributions in Different Beam- Offsets: Hodoscope Accepted True Dimuons $\phi_{q_{T}}$}
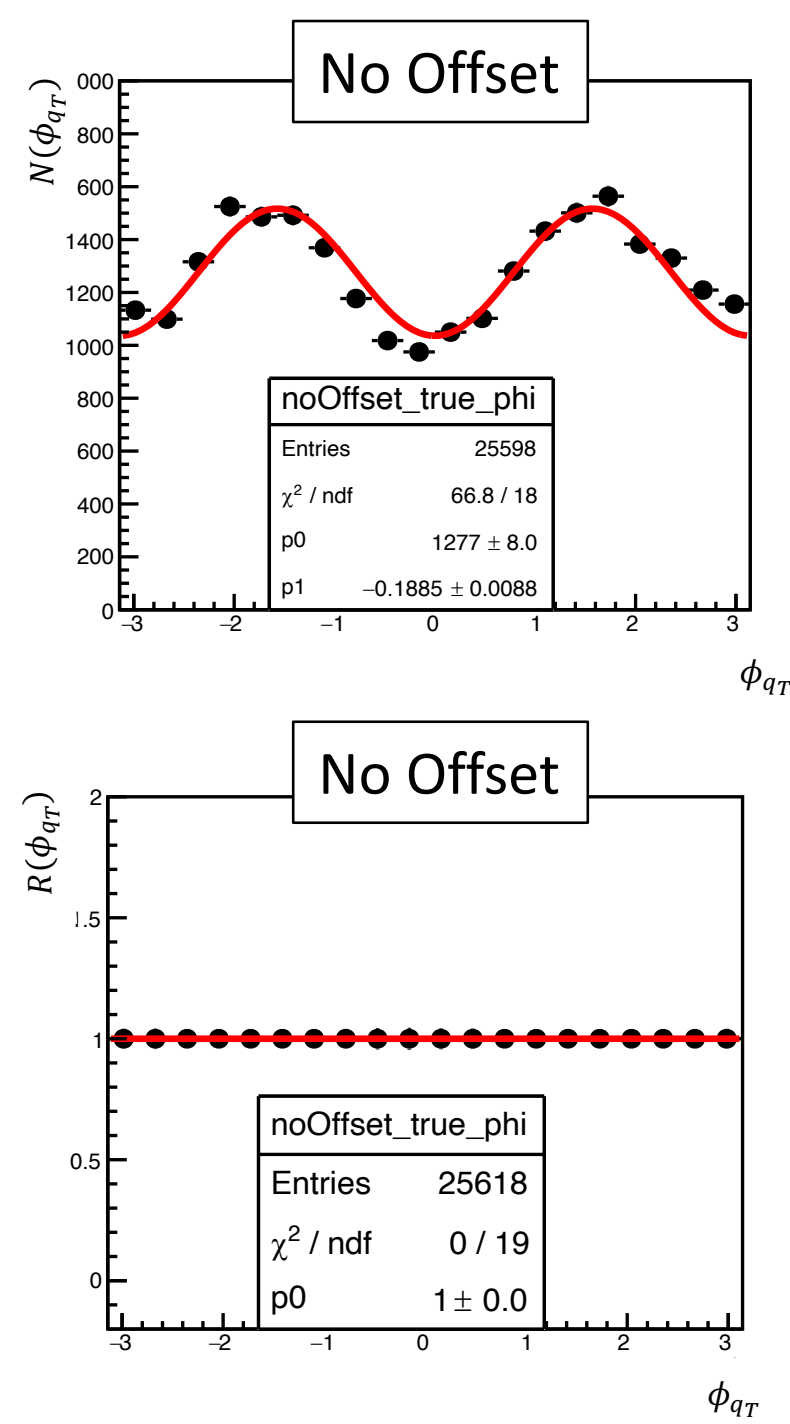
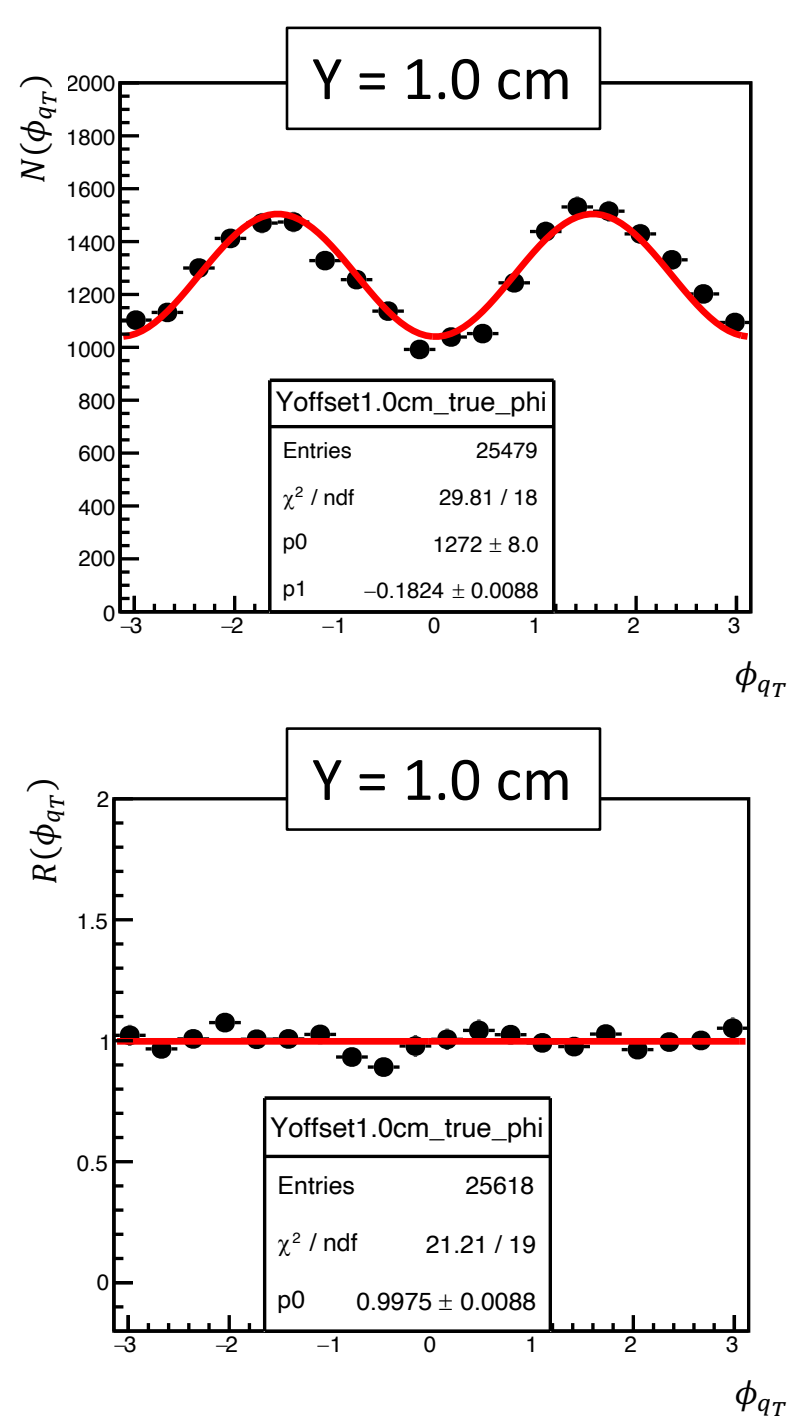
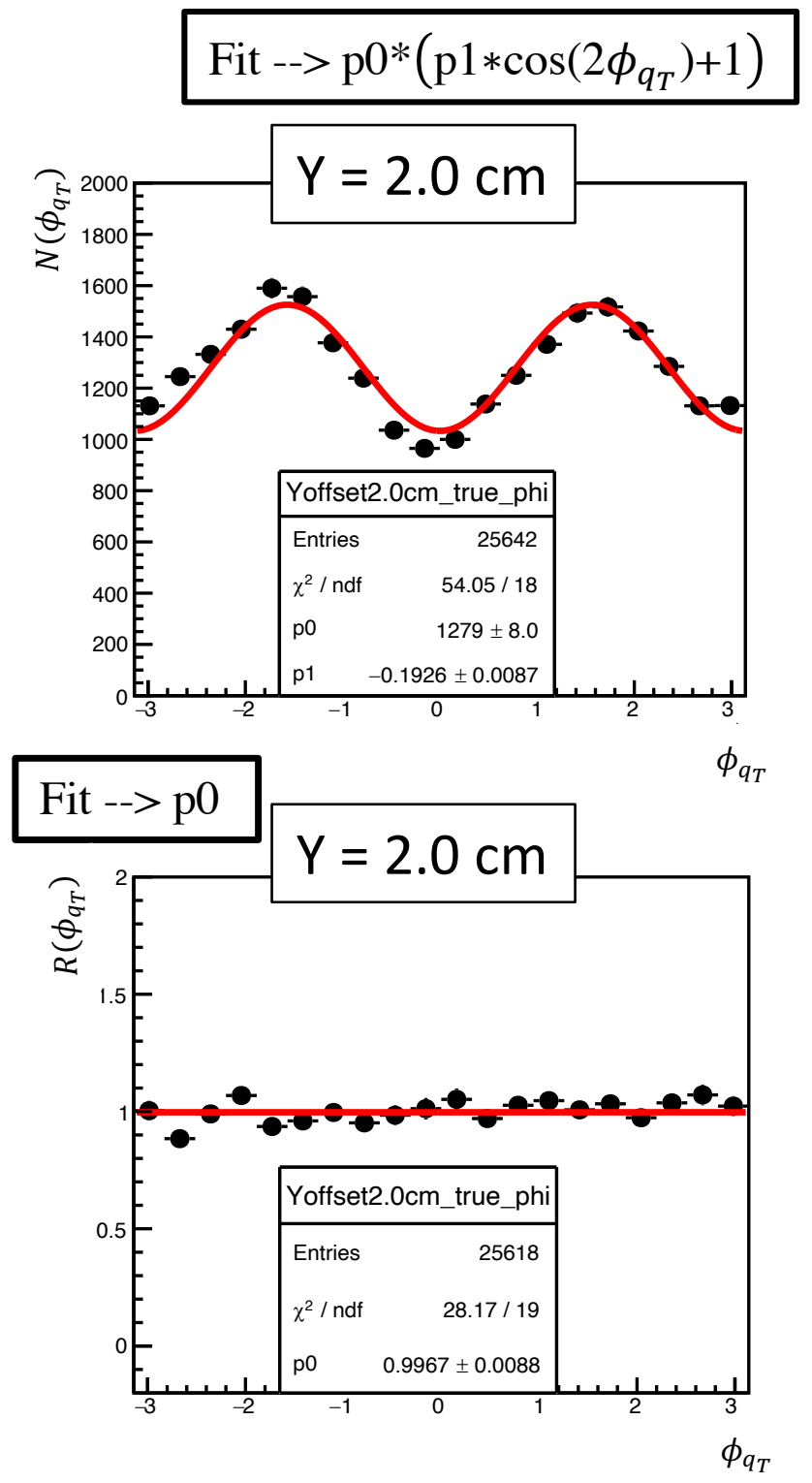


\section{Azimuthal Distributions in Different Beam-Offsets: Reconstructed $\phi_{q_{T}}$ When Offset is Unknown}
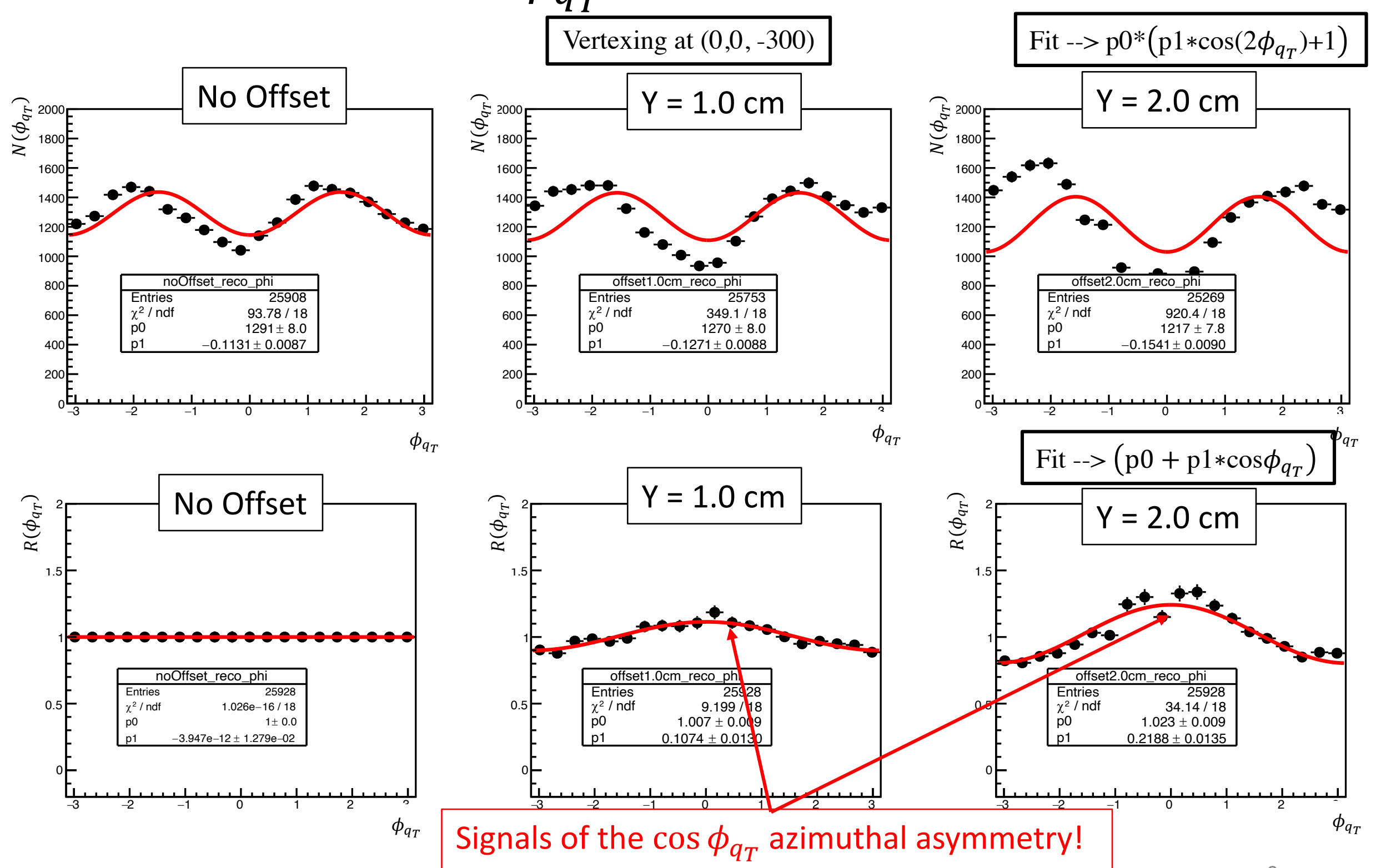

Beam-offset affect phi distributions if we did not know the offset information. 


\section{Azimuthal Distributions in Different Beam-Offsets: Reconstructed $\phi_{q_{T}}$ Ratio When Offset is Known}
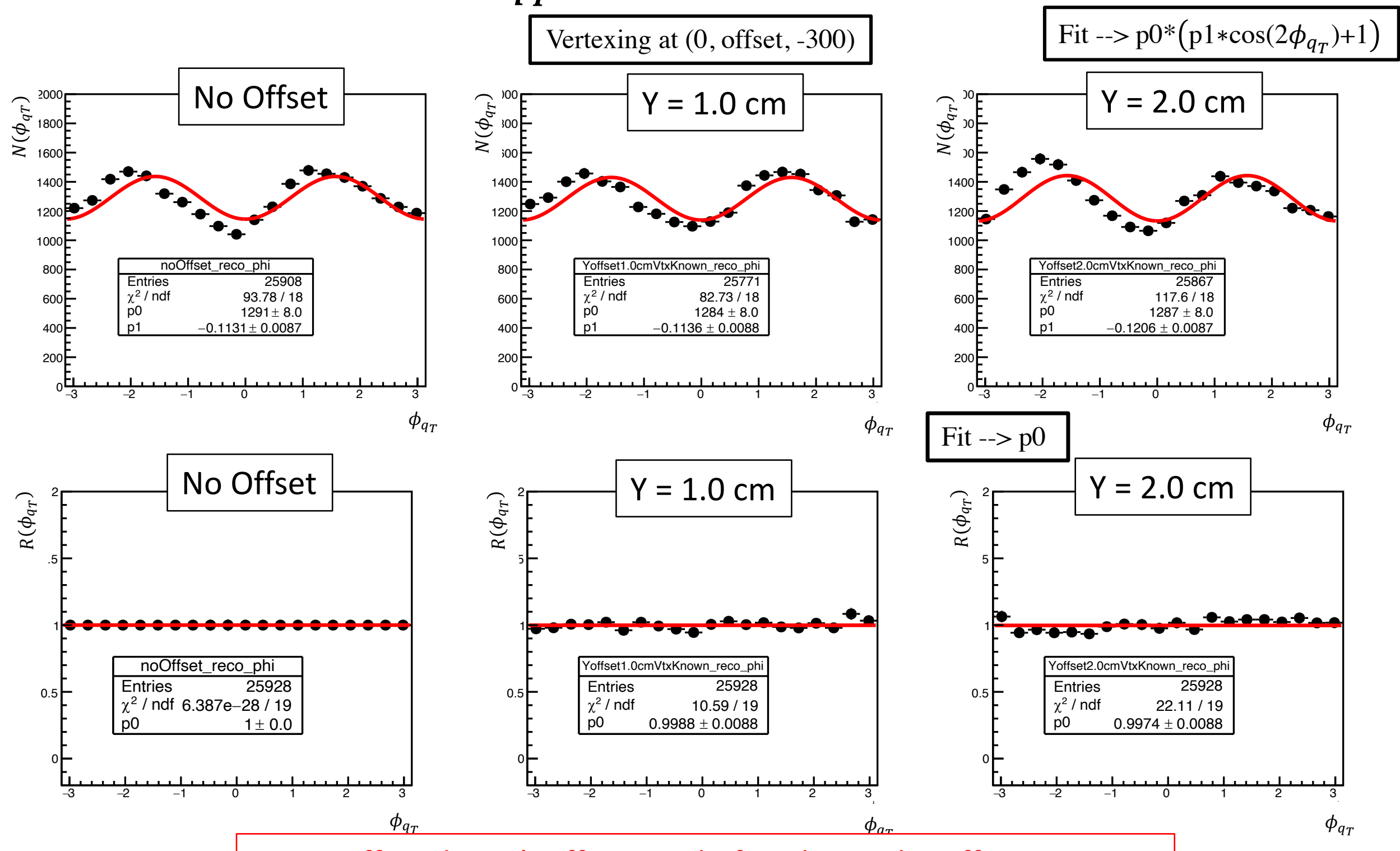

Beam-offset doesn't affect much if we knew the offset amount.
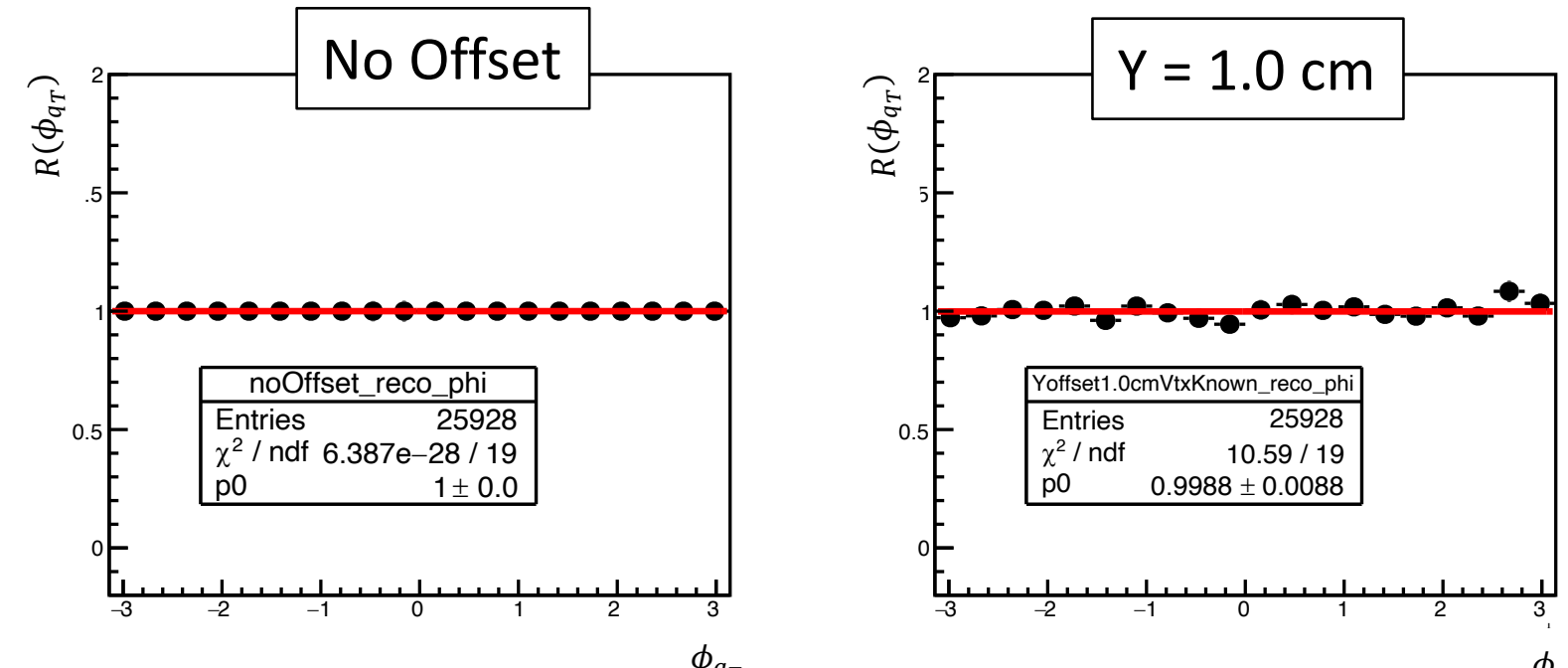


\section{Summary and Conclusions}

1. We have done a systematic study of measuring the fake azimuthal asymmetries that could be introduced due to the spectrometer.

2. We have found that the detector did not generate a false azimuthal asymmetry that could imitate the physics Sivers asymmetry.

3. From the beam-offset study we have found that the azimuthal distributions of dimuon phi will be distorted if the beam-offset was unknown, and the shape can be restored if we know the exact offset amount.

4. In SeaQuest the beam position measurement accuracy was about 1 $\mathrm{mm}$ and was stable during the data taking. We should not be sensitive to the beam-offset.

5. Beam angle and $x$ offset studies are going on. 